\title{
Simultaneous Bilateral Stenosis of the Vertebral Arteries Treated by Unilateral Decompression: A Case Report
}

\author{
Jin Hoon PARK, ${ }^{1}$ Seung Hoon YOU, ${ }^{1}$ Sung Woo ROH, ${ }^{2}$ In Seok HWANG, ${ }^{3}$ \\ and Sang-Youl LEE ${ }^{1}$ \\ ${ }^{1}$ Department of Neurological Surgery, Gangneung Asan Hospital, \\ University of Ulsan College of Medicine, Gangneung, Korea; \\ ${ }^{2}$ Department of Neurological Surgery, Asan Medical Center, \\ University of Ulsan College of Medicine, Seoul, Korea; \\ ${ }^{3}$ Department of Neurological Surgery, Baroseum Hospital, Daejeon, Korea
}

\begin{abstract}
A 56-year-old man presented with a 3-month history of progressive dizziness. His dizziness was aggravated when his head was rotated to the right side. Diagnostic angiography showed that a normal right-sided vertebral artery in the neutral position became an abnormal vertebral artery with two stenotic lesions at the C3-4 and C5-6 levels when the patient's head was turned to the right. A normal left-sided vertebral artery also showed a stenotic lesion at the $\mathrm{C} 2$ level when the patient's head was turned right. The axial dimensions of the bilateral vertebral arteries were similar. The patient was successfully treated with decompression of only one level (C5-6). We conclude that if a bilateral stenosis is found upon one directional head rotation and the bilateral vertebral arteries are similarly sized, a one-sided treatment may suffice.
\end{abstract}

Key words: vertebral artery compression, bow hunter's syndrome, dizziness, head rotation, angiography

\section{Introduction}

Symptomatic compression of the vertebral artery (VA) related to cervical spondylosis is rare. ${ }^{1-6)}$ Rotational VA occlusion syndrome, also called bow hunter's syndrome, results from mechanical occlusion or stenosis of the VA by surrounding structures, such as membranous or fibrous bands, a disc herniation, or osteophytes. ${ }^{7-11)}$ In most cases, symptoms appear on head extension or rotation to one side. The symptoms produced by this syndrome include ataxia, drop attacks, vertigo, nausea and vomiting, diplopia, dysarthria, tinnitus, and visual disturbances. ${ }^{8,12)}$

The unilateral occurrence of rotational VA occlusion syndrome has been reported several times and it is usually accompanied by hypoplasia of the contralateral VA or by poor collateral flow from the anterior cerebral circulation via the posterior communicating artery. ${ }^{2,5,13-17)}$ Only one report has described a case of bilateral dominant VA compression at the same level, which was treated bilaterally. ${ }^{8)}$ Another article has reported a case of bilateral angiographical VA stenosis at different levels, which was treated by unilateral decompression. ${ }^{18)}$

Received April 3, 2013; Accepted July 10, 2013
Here, we report a case of bilateral stenosis of three different levels that was successfully treated by decompression of only one level.

\section{Case Report}

A 56-year-old man presented with a 3-month history of progressive whirling-type dizziness. His dizziness was aggravated when his head was rotated to the right side. It was relieved immediately if his head adopted a neutral position. There was no neurological deficit, including nystagmus. Peripherally originating dizziness was also ruled out. Magnetic resonance imaging showed no sign of ischemic stroke. Computed tomography angiography revealed a bilateral bony spur at the C6-7 level and a normal finding of the VA. The axial dimensions of the bilateral VAs were similar (Fig. 1). Diagnostic angiography showed that a normal right-sided VA in the neutral position became an abnormal VA with two stenotic lesions at the C3-4 and C5-6 levels when the patient's head was turned to the right (Fig. 2). The normal left-sided VA also showed a stenotic lesion at the $\mathrm{C} 2$ level when the patient's head was turned to the right (Fig. 3). 


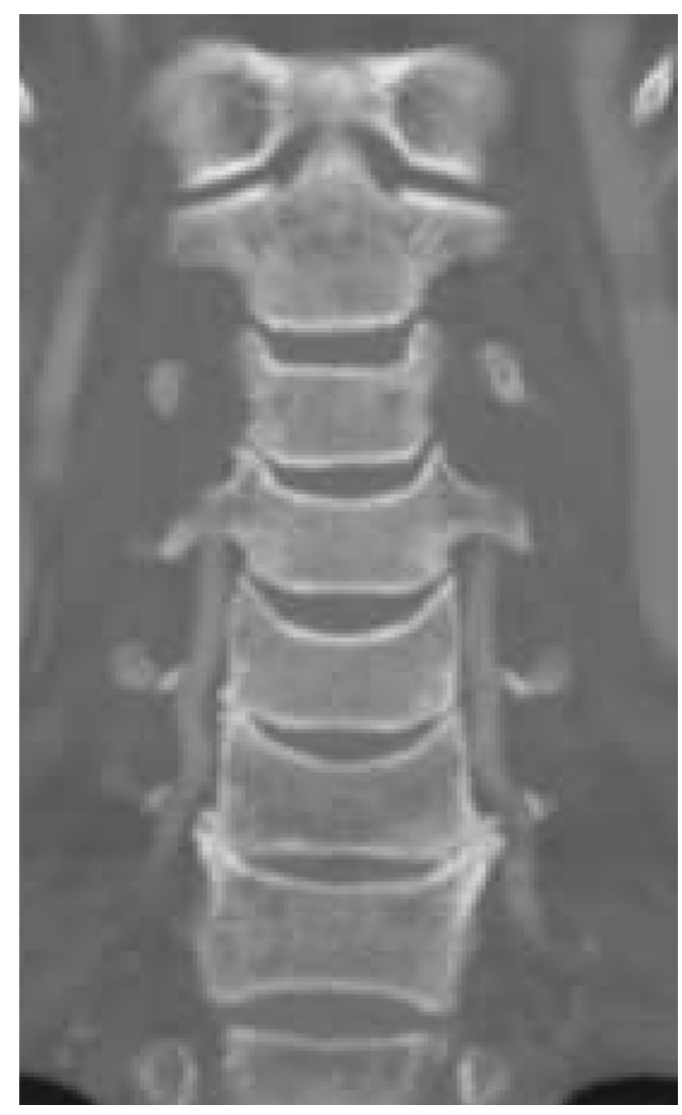

Fig. 1 Computed tomography angiography showing a rightsided osteophyte and linked VA at the C5-6 and C6-7 levels in the patient. The dimensions of the bilateral VAs were similar. VA: vertebral artery.

We reasoned that unilateral decompression of the VA at the C5 and C6 foramen could achieve a successful result because the dimensions of the bilateral VAs were similar. We also believed that if a right-sided lower level decompression was performed, the increased flow would affect the C3-4 level on the same side and the overall flow would be sufficient, even if mild mechanical stenosis remained at the C3-4 level. Nonetheless, we explained to the patient beforehand that he may subsequently require an additional second-stage surgery on the right side of the upper level.

The patient was placed in a supine position after general anesthesia. The usual right-sided Smith-Robinson approach was used and the longus colli muscle of the right side at the C5-6-7 level was detached with a monopolar coagulator. After the lateral tips of the right transverse processes of C5 and C6 were exposed, a retractor was applied. Once the transverse processes of C5 and C6 were unroofed with a 1.8-mm sized match head-shaped burr, the redundancy of the VA was checked. After the fibrous adhesion of the VA to the lateral side

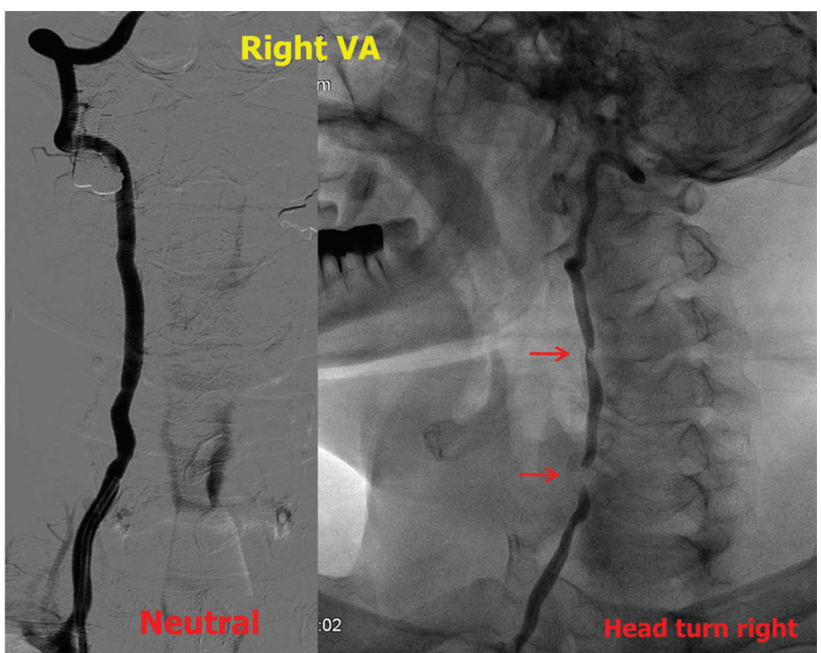

Fig. 2 A preoperative diagnostic angiography showing a normal right-sided VA in the neutral position that became an abnormal VA with two stenotic lesions at the C3-4 and C5-6 levels when the patient's head was turned to the right (arrows). VA: vertebral artery.

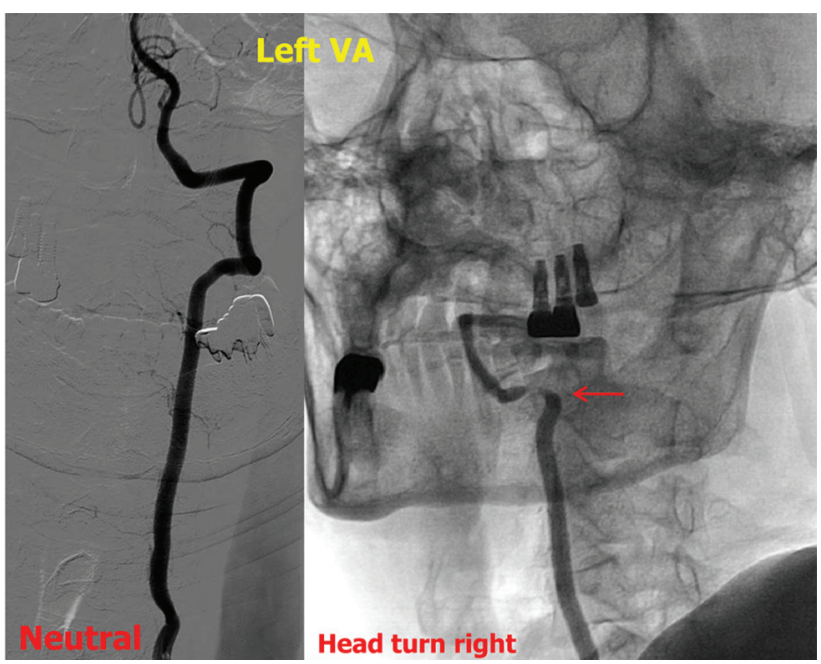

Fig. 3 A left-sided VA showing a stenotic lesion at the C2 level when the patient's head was turned to the right on preoperative diagnostic angiography (arrow). VA: vertebral artery.

of the C5-6 vertebral body was identified, decompression of the lateral side of the C5-6 was performed. The wound was closed when the free redundancy of the VA was identified. There were no abnormal events during the surgery.

After one day of an intensive care unit stay, the patient was satisfied with his improved symptoms. On postoperative day 7 , a follow-up angiography showed an improvement in the overall flow of the right VA 
when the patient's head was turned to the right (Fig. 4). Postoperative computed tomography angiography imaging demonstrated the removal of both the right uncovertebral joint at the C5-6 level and the roof of the C5 and 6 transverse foramen (Fig. 5).

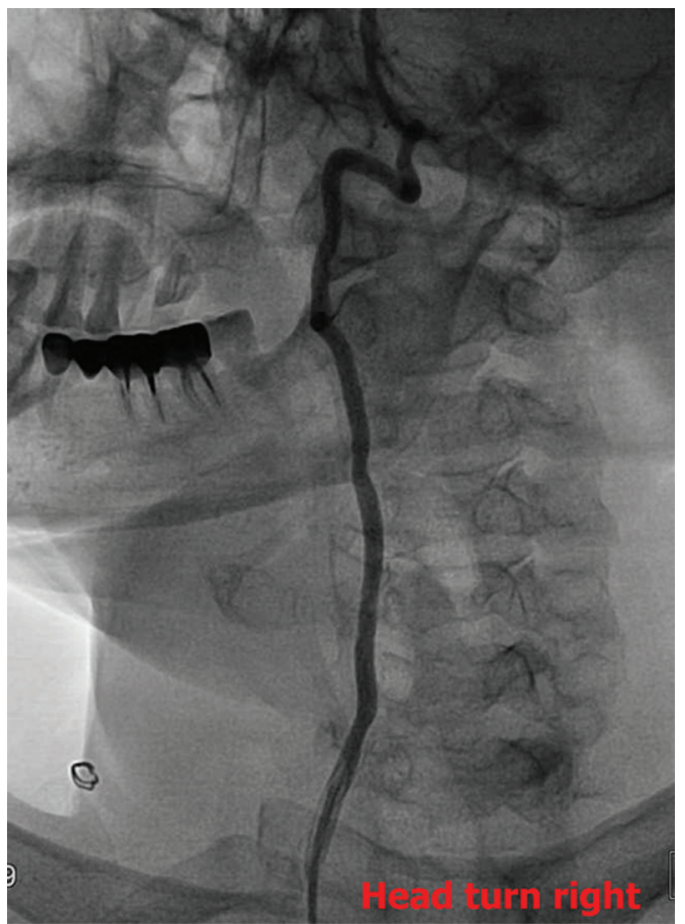

Fig. 4 A postoperative angiography showing that the overall flow of the right-sided VA was improved when the head was turned to the right. VA: vertebral artery.

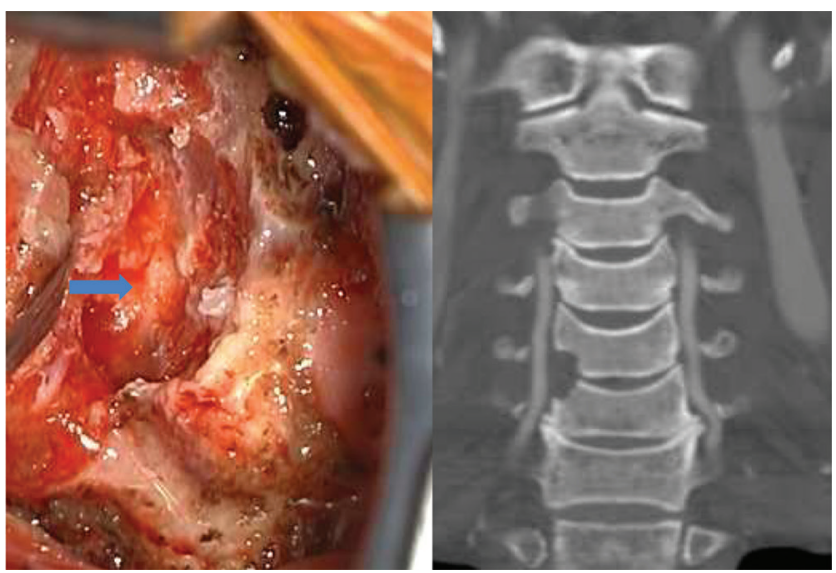

Fig. 5 An intraoperative image (left) and postoperative computed tomography angiography (right) showing a decompressed VA (arrow) and the removal of the uncovertebral joint and the roof of transverse foramen. VA: vertebral artery.

\section{Discussion}

A unilateral VA stenosis is normally asymptomatic if the other VA is able to compensate. A rotational VA syndrome may occur if the other VA is hypoplastic or has an anatomic variation. The symptoms may also be caused by the presence of occlusive fibrous tissue bands

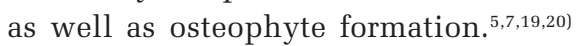

VA stenoses with lesions located above the C3 have a contralateral symptom presentation caused by stretching of the VA, but those located below the C3 have an ipsilateral presentation caused by compression. In stretching cases, surgical fixation seems to be the most reasonable treatment strategy, whereas in compression cases, decompression is more suitable. Although anterior decompression techniques have been reported, and the anterior approach may be preferred for a low cervical lesion, the posterior approach is preferred for a high cervical lesion.,14-16,21) For lower cervical decompression with the anterior approach, the removal of osteophytes and the roof of the transverse process of the upper vertebral body is emphasized, as in our present case. ${ }^{18,22,23)}$

Unusually, our patient simultaneously had two rightsided lower cervical compressions and one left-sided high cervical stretching. Accordingly, decompression of the right side was more appropriate than fusion surgery of the left, given the importance of the preservation of postoperative neck motion. In addition, we believed that if the blood flow were improved by decompression of the lower level, the increased blood flow at the lower cervical level would improve the flow on the right-sided C3-4 level.

Bilateral same level rotational VA compression successfully treated with bilateral decompression and fusion is extremely rare and only one case has been reported. ${ }^{8)}$ In that report, a patient with bilateral VA stenosis at the C4-5 level due to a bony spur complained of several symptoms related to VA insufficiency that were exacerbated upon rotation of his head to either side. Bilateral VA decompression and fusion surgery successfully treated the patient. ${ }^{8)}$ Our present patient differs from this earlier case on a number of points. First, our patient showed bilateral angiographic stenoses at different levels. Second, his symptoms and angiographic VA stenosis were aggravated only if the head was rotated to the right side. Third, we were able to achieve a successful result via decompression of the right-sided VA alone without fusion.

We surmised that a decompression of the right side alone would achieve a successful clinical result because unilateral VA stenosis does not usually cause neurological deterioration. We believed that stenosis of the lower level of the right side was induced by bony compression and that the upper level compression was related to VA stretching caused by membranous or fibrous bands. However, we were not certain that lower level decompression of the right side alone would give us a 
satisfactory clinical result and that a change in the flow of the lower level would correct the flow of the upper level. Accordingly, we explained to the patient before the surgery that there was a possibility of an additional second-stage surgery on the upper level of the right side. After surgery, the symptoms of the patient completely disappeared and additional surgery was not needed.

Two cases that are similar to ours have been reported. ${ }^{18,24)}$ In the report of Kawaguchi et al., ${ }^{18)}$ a right-sided C4-5 level VA stenosis and left-sided C1-2 level VA stenosis occurred when the head was rotated to the right. The patient was successfully treated with decompression of the transverse foramen and spur at the C4-5 level of the right side alone. The authors considered that the left-sided C1-2 VA stenosis was the only physiological finding. In addition, the crucial factor during the decompression of the lower level VA was osteophyte removal, as well as the removal of the transverse foramen of the upper vertebral body. In the report of Shinohara et al., ${ }^{24)}$ right-sided C3-4 level VA and left-sided C1-2 level VA stenoses occurred when the head was rotated to the right. The authors also successfully treated the patient with just right-sided decompression and fusion with an anterior approach. They suggested that an anterior decompression and fusion with osteophyte removal may be a wiser approach than arterial decompression or posterior fusion at the C1-2 level when faced with a two VA occlusive lesions. ${ }^{24)}$

We also agree with the opinions expressed in the papers of Kawaguchi et al., ${ }^{18)}$ and Shinohara et al., ${ }^{24)}$ that contralateral high level VA stenoses need not be treated because the one-sided patency of the VA is enough to ameliorate the symptoms, as shown here in our present case report. Another point is that low level decompression could change the blood flow dynamics, removing the need for high level decompression of the same side. Kawaguchi et al., ${ }^{18)}$ also suggested that fusion after decompression may better prevent hyperrotation and subluxation. We could achieve a successful result by decompression alone without fusion in our patient. Although there was no evidence of vertebrae instability during the short-term follow-up period, careful long-term follow-up is needed.

\section{Conclusion}

We successfully treated a patient showing bilateral stenoses at three different cervical levels with decompression of only one level. If bilateral stenoses are found in bilateral VAs of similar sizes, a one-sided treatment may be sufficient.

\section{Conflicts of Interest Disclosure}

The authors have no financial interest or ethical conflicts to disclose.
Jin Hoon Park and Seung Hoon You have equally contributed for this work.

\section{References}

1) Citow JS, Macdonald RL: Posterior decompression of the vertebral artery narrowed by cervical osteophyte: case report. Surg Neurol 51: 495-498; discussion 498-499, 1999

2) Fox MW, Piepgras DG, Bartleson JD: Anterolateral decompression of the atlantoaxial vertebral artery for symptomatic positional occlusion of the vertebral artery. Case report. J Neurosurg 83: 737-740, 1995

3) Kuether TA, Nesbit GM, Clark WM, Barnwell SL: Rotational vertebral artery occlusion: a mechanism of vertebrobasilar insufficiency. Neurosurgery 41: 427-432; discussion 432-433, 1997

4) Morimoto T, Kaido T, Uchiyama Y, Tokunaga H, Sakaki T, Iwasaki S: Rotational obstruction of nondominant vertebral artery and ischemia. Case report. J Neurosurg 85: 507-509, 1996

5) Velat GJ, Reavey-Cantwell JF, Ulm AJ, Lewis SB: Intraoperative dynamic angiography to detect resolution of Bow Hunter's syndrome: Technical case report. Surg Neurol 66: 420-423; discussion 423, 2006

6) Whitmore RG, Simon SL, Hurst RW, Nisenbaum HL, Kasner SE, Zager EL: Bow hunter's syndrome caused by accessory cervical ossification: posterolateral decompression and the use of intraoperative Doppler ultrasonography. Surg Neurol 67: 169-171, 2007

7) Darkhabani MZ, Thompson MC, Lazzaro MA, Taqi MA, Zaidat OO: Vertebral artery stenting for the treatment of bow hunter's syndrome: report of 4 cases. J Stroke Cerebrovasc Dis 21: 908. e1-5, 2012

8) Fleming JB, Vora TK, Harrigan MR: Rare case of bilateral vertebral artery stenosis caused by C4-5 spondylotic changes manifesting with bilateral bow hunter's syndrome. World Neurosurg 79: 799. E1-5, 2013

9) Hanakita J, Miyake H, Nagayasu S, Nishi S, Suzuki T: Angiographic examination and surgical treatment of bow hunter's stroke. Neurosurgery 23: 228-232, 1988

10) Ujifuku K, Hayashi K, Tsunoda K, Kitagawa N, Hayashi T, Suyama K, Nagata I: Positional vertebral artery compression and vertebrobasilar insufficiency due to a herniated cervical disc. J Neurosurg Spine 11: 326-329, 2009

11) Vates GE, Wang KC, Bonovich D, Dowd CF, Lawton MT: Bow hunter stroke caused by cervical disc herniation. Case report. J Neurosurg 96(1 Suppl): 90-93, 2002

12) Akar Z, Kafadar AM, Tanriover N, Dashti RS, Islak C, Kocer N, Kuday C: Rotational compression of the vertebral artery at the point of dural penetration. Case report. J Neurosurg 93: $300-303,2000$

13) Horowitz M, Jovin T, Balzar J, Welch W, Kassam A: Bow hunter's syndrome in the setting of contralateral vertebral artery stenosis: evaluation and treatment options. Spine 27: E495-498, 2002

14) Matsuyama T, Morimoto T, Sakaki T: Bow Hunter's stroke caused by a nondominant vertebral artery occlusion: case report. Neurosurgery 41: 1393-1395, 1997

15) Matsuyama T, Morimoto T, Sakaki T: Comparison of C1-2 posterior fusion and decompression of the vertebral artery 
in the treatment of bow hunter's stroke. J Neurosurg 86: 619-623, 1997

16) Seki $T$, Hida $K$, Akino M, Iwasaki Y: Anterior decompression of the atlantoaxial vertebral artery to treat bow hunter's stroke: technical case report. Neurosurgery 49: 1474-1476, 2001

17) Vilela MD, Goodkin R, Lundin DA, Newell DW: Rotational vertebrobasilar ischemia: hemodynamic assessment and surgical treatment. Neurosurgery 56: 36-43, 2005

18) Kawaguchi T, Fujita S, Hosoda K, Shibata Y, Iwakura M, Tamaki N: Rotational occlusion of the vertebral artery caused by transverse process hyperrotation and unilateral apophyseal joint subluxation. Case report. J Neurosurg 86: 1031-1035, 1997

19) Mapstone T, Spetzler RF: Vertebrobasilar insufficiency secondary to vertebral artery occlusion froma fibrous band. Case report. J Neurosurg 56: 581-583, 1982

20) Shimizu T, Waga S, Kojima T, Niwa S: Decompression of the vertebral artery for bow-hunter's stroke. Case report. J Neurosurg 69: 127-131, 1988

21) Kim K, Isu T, Morimoto D, Kominami S, Kobayashi S, Teramoto A: Anterior vertebral artery decompression with an ultra- sonic bone curette to treat bow hunter's syndrome. Acta Neurochir (Wien) 150: 301-303; discussion 303, 2008

22) Bulsara KR, Velez DA, Villavicencio A: Rotational vertebral artery insufficiency resulting from cervical spondylosis: case report and review of the literature. Surg Neurol 65: 625-627, 2006

23) Lee V, Riles TS, Stableford J, Berguer R: Two case presentations and surgical management of Bow Hunter's syndrome associated with bony abnormalities of the C7 vertebra. J Vasc Surg 53: 1381-1385, 2011

24) Shinohara N, Kohno K, Takeda S, Ohta S, Sakaki S: [A case of bow hunter's stroke caused by bilateral vertebral artery occlusive change on head rotation to the right.] No Shinkei Geka 26: 417-422, 1998 (Japanese)

Address reprint requests to: Sang-Youl Lee, MD, PhD, Department of Neurological Surgery, Gangneung Asan Hospital, University of Ulsan College of Medicine, 38 Bangdong-gil, Sacheon-myeon, Gangneung 210-711, Korea. e-mail: grandblue@gnah.co.kr 